\title{
THE $h$-VECTOR OF A GORENSTEIN CODIMENSION THREE DOMAIN
}

\author{
E. DE NEGRI AND G. VALLA
}

Let $k$ be an infinite field and $A$ a standard $G$-algebra. This means that there exists a positive integer $n$ such that $A=R / I$ where $R$ is the polynomial ring $R:=k\left[X_{1}, \ldots, X_{n}\right]$ and $I$ is an homogeneous ideal of $R$. Thus the additive group of $A$ has a direct sum decomposition $A=\bigoplus A_{\imath}$ where $A_{i} A_{j} \subseteq A_{i+j}$. Hence, for every $t \geq 0, A_{t}$ is a finite-dimensional vector space over $k$. The Hilbert Function of $A$ is defined by

$$
H_{A}(t):=\operatorname{dim}_{k}\left(A_{t}\right), \quad t \geq 0 .
$$

The generating function of this numerical function is the formal power series

$$
P_{A}(z):=\sum_{t \geq 0} H_{A}(t) z^{t}
$$

As a consequence of the Hilbert-Serre theorem we can write

$$
P_{A}(z)=h_{A}(z) /(1-z)^{d}
$$

where $h_{A}(z) \in \mathbf{Z}[z]$ is a polynomial with integer coefficients such that $h_{A}(1) \neq 0$. Moreover $d$ is the Krull dimension of the ring $A$.

The polynomial $h_{A}(z)$ is called the $h$-polynomial of $A$; if $h_{A}(z)=1+a_{1} z+$ $\cdots+a_{s} z^{s}$ with $a_{s} \neq 0$, then we say that the vector $\left(1, a_{1}, \ldots, a_{s}\right)$ is the $h$-vector of $A$. It is clear that the $h$-vector of $A$ together with its Krull dimension determines the Hilbert Function of $A$ and conversely.

A classical result of Macaulay gives an explicit numerical characterization of the admissible numerical functions, i.e. of the functions $H: \mathbf{N} \rightarrow \mathbf{N}$ which are the Hilbert Function of some standard $\mathrm{G}$-algebra $A$. This result proved in $[\mathrm{M}]$ has been recently revisited by Stanley in [S]. One can easily find similar characterizations for reduced or Cohen-Macaulay G-algebras (see [GMR] and [S]).

Received August 3, 1993.

Revised June 14, 1994

The second author was partially supported by M.P.I. (Italy). 
The problem is much more difficult if one deals with Cohen-Macaulay integral domains. Only in the codimension two case we have a complete answer given by Peskine and Gruson in [GP] using deep geometric methods.

If we come to the Gorenstein case, very little is known. In [S] Stanley used the structure theorem of Buchsbaum and Eisenbud for codimension three Gorenstein ideals in order to give a complete characterization of the corresponding $h$-vector. It is then natural to ask for other restrictions on the $h$-vector of a Gorenstein codimension three $\mathrm{G}$-algebra $A$ if we assume moreover that $A$ is an integral domain.

In this paper we answer this question by using a lifting theorem recently proved in [HTV], which asserts that every codimension three homogeneous Gorenstein ideal with degree matrix verifying certain numerical conditions can be lifted to a codimension three Gorenstein prime ideal (see Lemma 3).

Let us fix some notations. If $h(z) \in \mathbf{Z}[z]$ we define its difference $\Delta h(z)$ by

$$
\Delta h(z):=h(z)(1-z) .
$$

If $h(z)$ is a multiple of $1-z$ then we define its sum $\sum h(z)$ by

$$
\sum h(z):=\frac{h(z)}{(1-z)} .
$$

If we have $h(z)=\sum_{i=0}^{s} a_{i} z^{\imath}$, then it is clear that $\Delta h(z)=\sum_{i=0}^{s+1} b_{i} z^{i}$ where

$$
b_{i}=a_{\imath}-a_{\imath-1}, \quad i=0, \ldots, s+1 .
$$

Moreover if $h(z)$ is a multiple of $1-z$ then $\sum h(z):=\sum_{l=0}^{s-1} c_{\imath} z^{2}$ where

$$
c_{i}=\sum_{j=0}^{i} a_{i}, \quad i=0, \ldots, s-1 .
$$

We say that the polynomial $h(z)=\sum_{i=0}^{s} a_{i} z^{\imath} \in \mathbf{Z}[z]$ is $s$-symmetric if $a_{i}=$ $a_{s-i}$ for every $i=0, \ldots, s$, while we say that it is $s$-antisymmetric if $a_{i}=-a_{s-i}$ for every $i=0, \ldots, s$.

It is easy to see that if $h(z)$ is $s$-symmetric then $\Delta h(z)$ is $(s+1)$ antisymmetric, while if $h(z)$ is a multiple of $1-z$ and is $s$-antisymmetric then $\sum h(z)$ is $(s-1)$-symmetric.

Let now $I$ be a codimension three homogeneous Gorenstein ideal of the polynomial ring $R:=k\left[X_{1}, \ldots, X_{n}\right]$. By the structure theorem of Buchsbaum and Eisenbud [BE], there exists an integer $g \geq 1$ such that $I$ is minimally generated by 
the $2 g$-pfaffians of a $(2 g+1) \times(2 g+1)$ skew-symmetric matrix $\left(F_{i j}\right)$ with homogeneous entries. We denote by $p_{t}$ the pfaffian of the skew-symmetric matrix which is obtained from $\left(F_{\imath j}\right)$ by deleting the $i$-th row and the $i$-th column. Then $I=\left(p_{1}, \ldots, p_{2 g+1}\right)$. Let $a_{1}, \ldots, a_{2 g+1}$ be the degrees of these pfaffians. Since $R / I$ is Gorenstein, it has a self-dual free homogeneous resolution as an $R$-module:

$$
0 \rightarrow R(-c) \rightarrow \bigoplus_{i=1}^{2 g+1} R\left(-b_{i}\right) \rightarrow \bigoplus_{i=1}^{2 g+1} R\left(-a_{i}\right) \rightarrow R \rightarrow R / I \rightarrow 0
$$

We may assume that

$$
2 \leq a_{1} \leq a_{2} \leq \cdots \leq a_{2 g+1} .
$$

Since the resolution is self-dual we get

$$
b_{i}=c-a_{i}, \quad i=1, \ldots, 2 g+1 .
$$

From the additivity of the Poincaré series, we can write

$$
\begin{aligned}
P_{R / I}(z)=P_{R}(z)-\sum_{i=1}^{2 g+1} P_{R\left(-a_{i}\right)}(z)+\sum_{i=1}^{2 g+1} P_{R\left(-b_{i}\right)}(z)-P_{R(-c)}(z)= \\
=\frac{1-\sum_{i=1}^{2 g+1} z^{a_{i}}+\sum_{l=1}^{2 g+1} z^{b_{i}}-z^{c}}{(1-z)^{n}} .
\end{aligned}
$$

Since $\operatorname{dim}(R / I)=n-3$, we have

$$
h_{A}(z)=\frac{f(z)}{(1-z)^{3}}
$$

where

$$
f(z):=1-\sum_{i=1}^{2 g+1} z^{a_{i}}+\sum_{i=1}^{2 g+1} z^{b_{i}}-z^{c}
$$

is a multiple of $(1-z)^{3}$. This means that its derivative vanishes at 1 so that

$$
-\sum_{i=1}^{2 g+1} a_{i}+\sum_{i=1}^{2 g+1} b_{i}-c=0 .
$$

Using the fact that $b_{i}=c-a_{i}$, we get

$$
c=\frac{1}{g} \sum_{i=1}^{2 g+1} a_{i}
$$

This proves that the degrees of a minimal set of homogeneous generators of a Gorenstein codimension three ideal completely determine the numerical characters 
of the resolution.

We consider the matrix $\left(u_{i j}\right)$ where we let

$$
u_{\imath}:=b_{\imath}-a_{j}, \quad i, j=1, \ldots, 2 g+1 .
$$

This matrix is then uniquely determined by $I$ and is called the degree matrix of $I$. It is clear that $\left(u_{\imath}\right)$ is a symmetric matrix and

$$
\operatorname{deg}\left(F_{i j}\right)=b_{i}-a_{j}=u_{i j}, \quad i, j=1, \ldots, 2 g+1 .
$$

Since the resolution is minimal, this implies that $F_{i j}=0$ if $u_{i j} \leq 0$.

The degree matrix of $I$ verifies the following conditions:

(a) $u_{i j} \geq u_{s t}$ for $i \leq s$ and $j \leq t$.

(b) $u_{i j}+u_{s t}=u_{i t}+u_{s,}$ for every $i, j, s$ and $t$.

(c) $u_{i j}>0$ for all $i$ and $j$ such that $i+j=2 g+3$.

The first two conditions are obvious. As for (c), if $u_{r, 2 g+3-r} \leq 0$, then by (a) $u_{i j} \leq 0$ for every $i \geq r$ and $j \geq 2 g+3-r$. This implies that $F_{i j}=0$ for the same indexes. But then $p_{1}=0$, a contradiction to the minimality of the resolution.

We remark that condition a) above can be visualized by observing that it has the following meaning: the entries of the matrix do not decrease if we move up or left inside the matrix.

Further if $c \leq b_{1}=c-a_{1}$ then $a_{1} \leq 0$, a contradiction. Hence we certainly have

$$
\begin{aligned}
c>b_{1} \geq b_{2} & \geq b_{3} \geq \cdots \geq b_{2 g+1} \\
\vee & \vee \\
a_{2 g+1} & \geq a_{2 g} \geq \cdots \geq a_{2} \geq a_{1} \geq 2 .
\end{aligned}
$$

The converse of the above result is also true: we insert here a proof for the sake of completeness.

Lemma 1. Let $2 \leq a_{1} \leq \cdots \leq a_{2 g+1}$ be integers such that for some integer $c$ we have $\mathrm{cg}=\sum_{i=1}^{2 g+1} a_{i}$. For every $i=1, \ldots, 2 g+1$, let $b_{i}:=c-a_{i}$. If the matrix $\left(u_{i j}\right):=\left(b_{i}-a_{j}\right)$, which certainly verifies conditions (a) and (b), also verifies the above condition (c), then there exists a codimension three Gorenstein ideal $I$ in $R=$ $k[X, Y, Z]$ such that $R / I$ has a minimal free resolution

$$
0 \rightarrow R(-c) \rightarrow \bigoplus_{i=1}^{2 g+1} R\left(-b_{i}\right) \rightarrow \bigoplus_{i=1}^{2 g+1} R\left(-a_{i}\right) \rightarrow R \rightarrow R / I \rightarrow 0 .
$$

In particular I has degree matrix $\left(u_{i j}\right)$. 
Proof. In the polynomial ring $k[X, Y, Z]$ let $I$ be the ideal generated by the $2 g \times 2 g$ pfaffians of the skew-symmetric matrix $\left(F_{\imath j}\right)$ where

$$
\begin{cases}F_{i, 2 g+1-i}=X^{u_{i, 2 g+1-i}}, & i=1, \ldots, g, \\ F_{i, 2 g+1-i}=-X^{u_{i, 2 g+1-i}}, & i=g+1, \ldots, 2 g, \\ F_{i, 2 g+2-i}=Y^{u_{i, 2 g+2-i}}, & i=1, \ldots, g, \\ F_{i, 2 g+2-i}=-Y^{u_{i, 2 g+2-i}}, & i=g+2, \ldots, 2 g+1, \\ F_{i, 2 g+3-\imath}=Z^{u_{i, 2 g+3-i}}, & i=2, \ldots, g+1, \\ F_{i, 2 g+3-i}=-Z^{u_{i, 2 g+3-i},}, & i=g+2, \ldots, 2 g+1, \\ 0 & \text { otherwise. }\end{cases}
$$

We remark that since the matrix $\left(u_{i j}\right)$ verifies the condition c) all the exponents above are positive integers.

Furthermore, in order to get a homogeneous matrix, we assign degree $u_{i j}$ to the zero on the $i$-th row and $j$-th column. It is easy to see that

$$
p_{1}=Z^{\Sigma_{i=2}^{g+1} u_{i, 2 g+3-1}}, \quad p_{2 g+1}=X^{\Sigma_{i=1}^{g} u_{i, 2 g+1-i}}, p_{g+1}=Y^{\Sigma_{i=1}^{g} u_{i, 2 g+2-i}}+f(X, Y, Z)
$$

where $f(X, Y, Z) \in(X, Z)$. This means that $I$ is a codimension three ideal which is Gorenstein since it is generated by the pfaffians of a skew-symmetric matrix.

Moreover, since the determinant of a skew-symmetric matrix is the square of the pfaffian, we have

$$
\operatorname{deg}\left(p_{\imath}\right)=\frac{\sum_{\jmath \neq i} u_{j j}}{2}=\frac{\sum_{j \neq i}\left(c-2 a_{j}\right)}{2}=g c-\sum_{j \neq i} a_{j}=a_{\imath} .
$$

The conclusion then follows since we have seen that the degrees of a minimal set of homogeneous generators of a codimension three Gorenstein ideal completely determine the other numerical characters of the resolution.

If we assume now that the codimension three Gorenstein ideal is prime, then we have a stronger condition on the degree matrix. This is the content of the following result proved in [HTV], Lemma 5.1.

Lemma 2. Let $I \subseteq R=k\left[X_{1}, \ldots, X_{n}\right]$ be a codimension three homogeneous Gorenstein prime ideal with degree matrix $\left(u_{\imath}\right)$. If $g \geq 2$, then

$$
u_{\imath, 2 g+4-\imath}>0, \quad i=3, \ldots, g+1 \text {. }
$$


Proof. If $u_{t, 2 g+4-t} \leq 0$ for some $t$ such that $3 \leq t \leq g+1$, then $u_{\imath} \leq 0$ for every $i \geq t$ and $j \geq 2 g+4-t$, so that $F_{i j}=0$ for the same indexes (here, as before, $F_{\imath j}$ are the entries of the skew-symmetric matrix in the resolution of $R / I$ ). This implies that the $2(g+2-t)$-pfaffian obtained from the matrix $\left(F_{i j}\right)$ by deleting the first $t-1$ and the last $t-2$ rows and columns, is a common factor of $p_{1}$ and $p_{2}$. A contradiction.

We remark here that if we have $u_{i, 2 g+4-i}>0$ for $i=3, \ldots, g+1$ then, by the symmetry of the matrix $\left(u_{i j}\right)$, we also have $u_{i, 2 g+4-i}>0$ for $i=g+3, \ldots$, $2 g+1$. Thus on the diagonal where $i+j=2 g+4$ all the entries of the matrix $\left(u_{i j}\right)$ are positive integers except, possibly, for $u_{g+2, g+2}$.

Further it is clear that, if $g \geq 2$, then a degree matrix such that $u_{i, 2 g+4-i}>0$ for $i=3, \ldots, g+1$ verifies also condition c) above, namely $u_{i, 2 g+3-i}>0$ for every $i=2, \ldots, 2 g+1$. This because we can express this condition by saying that all the entries on the $(2 g+4)$-diagonal are positive and remark that for every element of the $(2 g+3)$-diagonal we can find an element on the $(2 g+4)$ diagonal which is right or below the given element and is different from $u_{g+2, g+2}$.

The following less trivial result is the lifting theorem we referred to in the introduction.

Let $I \subseteq R=k\left[X_{1}, \ldots, X_{n}\right]$ be an homogeneous ideal. We say that the ideal $I$ can be lifted to an ideal $J \subseteq S=k\left[X_{1}, \ldots, X_{m}\right], m \geq n$, if there exist $r=m-n$ linear forms $l_{1}, \ldots, l_{r} \in S$ such that:

a) $l_{1}, \ldots, l_{r}$ form a regular sequence $\bmod J$.

b) In the canonical isomorphism

$$
S /\left(l_{1}, \ldots, l_{r}\right) S \simeq R
$$

the ideal $\left(J+\left(l_{1}, \ldots, l_{r}\right) S\right) /\left(l_{1}, \ldots, l_{r}\right) S$ corresponds to $I$.

It is clear that if the ideal $I$ can be lifted to the ideal $J$, then

$$
P_{R / I}(z)=(1-z)^{m-n} P_{S / J}(z) .
$$

In particular they share the same $h$-polynomial.

Lemma 3. Let $I \subseteq R=k\left[X_{1}, \ldots, X_{n}\right]$ be a codimension three homogeneous Gorenstein ideal. Let us assume that either $g=1$ or $g \geq 2$ and the degree matrix $\left(u_{i j}\right)$ of I satisfies the condition

$$
u_{i, 2 g+4-i}>0
$$


for every $i=3, \ldots, g+1$. Then $I$ can be lifted to a codimension three Gorenstein prime ideal $J \subseteq S=k\left[X_{1}, \ldots, X_{m}\right]$, for some integer $m \geq n$.

A proof of this crucial result can be found in [HTV], Lemma 5.5.

Now let $h(z)=1+3 z+h_{2} z^{2}+\cdots+h_{s} z^{s}$ be a polynomial in $\mathbf{Z}[z]$ such that $h_{s} \neq 0$. The integer

$$
a:=\min \left\{t \mid h_{t} \neq\left(\begin{array}{c}
t+2 \\
2
\end{array}\right)\right\}
$$

is called the initial degree of $h(z)$. It is clear that $2 \leq a \leq s+1$. In the following, for a rational number $q$, we denote by $[q]$ its integer part.

LEMMA 4. If the polynomial $h(z)$ is s-symmetric, then

$$
2 \leq a \leq\left[\frac{s}{2}\right]+1
$$

Proof. If $2 a-2>s$, then $s-a+2<a$. This implies $h_{s-a+2}=$ $\left(\begin{array}{c}s-a+4 \\ 2\end{array}\right)$, hence, by the symmetry,

$$
\left(\begin{array}{l}
a \\
2
\end{array}\right)=h_{a-2}=\left(\begin{array}{c}
s-a+4 \\
2
\end{array}\right) .
$$

It follows that $s-a+4=a$. If $s$ is odd, this is a contradiction. If $s$ is even, say $s=2 t$, then $a=t+2$, hence

$$
\left(\begin{array}{c}
t+1 \\
2
\end{array}\right)=h_{t-1}=h_{t+1}=\left(\begin{array}{c}
t+3 \\
2
\end{array}\right),
$$

a contradiction. Hence $a \leq \frac{s}{2}+1$ and the conclusion follows.

In the following we will often use the trivial inequalities:

$$
s-1 \leq 2\left[\frac{s}{2}\right] \leq s .
$$

Given a polynomial $h(z)=1+3 z+h_{2} z^{2}+\cdots+h_{s} z^{s} \in \mathbf{Z}[z]$ such that $h_{s} \neq 0$, we denote by $a$ its initial degree and also we let 


$$
\sum_{t=0}^{s+2} q_{t} z^{t}:=h(z)(1-z)^{2}=\Delta^{2} h(z) .
$$

We can now prove the main result of this paper.

THEOREM 5. Given the polynomial $h(z)=1+3 z+h_{2} z^{2}+\cdots+h_{s} z^{s} \in$ $\mathbf{Z}[z]$ with $h_{s} \neq 0$, there exists a codimension three Gorenstein $G$-domain which has $h(z)$ as $h$-polynomial if and only if the following conditions are satisfied:

a) $h(z)$ is s-symmetric.

b) $q_{t} \leq 0$ for every $t$ such that $a \leq t \leq\left[\frac{s}{2}\right]+1$.

c) It does not happen that $q_{t}<0, q_{v}=0$ and $q_{r}<0$ with $a \leq t<v<r$ $\leq\left[\frac{s}{2}\right]+1$

Proof. Let us assume first that $h(z)$ is the $h$-polynomial of a Gorenstein $G$-domain $A$. Then it is well known that $h(z)$ is $s$-symmetric (see [S], Theorem 4.1). Let

$$
0 \rightarrow R(-c) \rightarrow \bigoplus_{i=1}^{2 g+1} R\left(-b_{i}\right) \rightarrow \bigoplus_{i=1}^{2 g+1} R\left(-a_{i}\right) \rightarrow R \rightarrow R / I \rightarrow 0
$$

be a graded free resolution of $A=R / I$, where we assume that

$$
a_{1} \leq a_{2} \leq \cdots \leq a_{2 g+1} .
$$

As we have seen before we have

$$
c=\frac{1}{g} \sum_{i=1}^{2 g+1} a_{\imath}, \quad b_{\imath}=c-a_{\imath}, i=1, \ldots, 2 g+1
$$

and

$$
h(z)=\frac{f(z)}{(1-z)^{3}}
$$

where we let

$$
f(z)=1-\sum_{i=1}^{2 g+1} z^{a_{i}}+\sum_{i=1}^{2 g+1} z^{b_{i}}-z^{c}
$$

Since

$$
h(z)=\left(1-\sum_{i=1}^{2 g+1} z^{a_{i}}+\sum_{i=1}^{2 g+1} z^{b_{i}}-z^{c}\right)\left(\sum_{t \geq 0}\left(\begin{array}{c}
t+2 \\
2
\end{array}\right) z^{t}\right)
$$


and

$$
a_{1}=\min \left\{a_{\imath}, b_{i}\right\}_{i=1, \ldots, 2 g+1},
$$

we have

$$
a=a_{1}
$$

Since, as we have seen before,

$$
c>a_{i}, b_{i}, \quad i=1, \ldots, 2 g+1,
$$

we also have

$$
c=s+3 \text {. }
$$

Now

$$
\sum_{t=0}^{s+2} q_{t} z^{t}:=h(z)(1-z)^{2}=\frac{1-\sum_{i=1}^{2 g+1} z^{a_{i}}+\sum_{l=1}^{2 g+1} z^{b_{i}}-z^{c}}{1-z}=\sum f(z) .
$$

From this we get

$$
q_{t}=1+\#\left\{m \mid b_{m} \leq t\right\}-\#\left\{m \mid a_{m} \leq t\right\}, \quad t=0 \ldots, s+2 .
$$

To better visualize our argument, we recall that, no matter $I$ is prime or not, we have:

$$
\begin{aligned}
c>b_{1} \geq b_{2} & \geq b_{3} \geq \cdots \geq b_{2 g+1} \\
\vee & \vee \\
a_{2 g+1} & \geq a_{2 g} \geq \cdots \geq a_{2} \geq a_{1} \geq 2 .
\end{aligned}
$$

We need also to remark that

$$
c-b_{1}=a_{1} \leq a_{2}<b_{2 g+1} \leq b_{1}
$$

so that

$$
\left[\frac{s}{2}\right]+1=\left[\frac{s+2}{2}\right]=\left[\frac{c-1}{2}\right] \leq\left[\frac{c}{2}\right] \leq \frac{c}{2}<b_{1}
$$

We prove now that condition b) holds.

Let $t$ be an integer such that

$$
a \leq t \leq\left[\frac{s}{2}\right]+1
$$

Then, by the above inequality, $a \leq t<b_{1}$. We have two possibilities: either 
$t<b_{2 g+1}$ or $b_{2 g+1} \leq t$.

In the first case since $a=a_{1} \leq t<b_{2 g+1}$, we immediately get

$$
\left\{m \mid b_{m} \leq t\right\}=\varnothing \text {, }
$$

and

$$
\left\{m \mid a_{m} \leq t\right\} \supseteq\{1\}
$$

This implies by $(*)$

$$
q_{t} \leq 1-1=0
$$

as wanted.

In the second case we have $b_{2 g+1} \leq t<b_{1}$, and we can find an integer $r$ such that

$$
2 \leq r \leq 2 g+1, \quad b_{r} \leq t<b_{r-1} \text {. }
$$

Hence

$$
a_{2 g+3-r}<b_{r} \leq t<b_{r-1}
$$

and we get

$$
\begin{aligned}
& \left\{m \mid b_{m} \leq t\right\}=\{r, r+1, \ldots, 2 g+1\}, \\
& \left\{m \mid a_{m} \leq t\right\} \supseteq\{1,2, \ldots, 2 g+3-r\} .
\end{aligned}
$$

From $(*)$ we get

$$
q_{t} \leq 1+(2 g+1-r+1)-(2 g+3-r)=0 .
$$

This proves $\mathrm{b}$ ).

We remark that, up to this point, we did not use the primality assumption.

Let us come to the last statement. By contradiction, let

$$
q_{t}<0, \quad q_{v}=0, \quad q_{r}<0
$$

with $a \leq t<v<r \leq\left[\frac{s}{2}\right]+1$.

Under this assumption we claim that

$$
b_{2 g+1} \leq v \leq a_{2 g+1}<b_{2} .
$$

The first inequality comes from the fact that $q_{t}<0$ and $q_{v}=0$, hence we need at 
least one $b_{\text {i }}$ 's to get a positive contribution in the sum in $(*)$. The second inequality follows from the same argument due to the fact that $q_{v}=0$ and $q_{r}<0$.

The claim implies that we can find an integer $d$ such that

$$
b_{d+1} \leq v<b_{d} .
$$

In the case $g=1$, we have

$$
b_{3} \leq v \leq a_{3}<b_{2},
$$

hence $d=2$ and either

$$
a_{2 g+3-d}=a_{3}=v<b_{2}=b_{d},
$$

or

$$
b_{g+2}=b_{3} \leq v<a_{3}=a_{g+2} .
$$

If $g \geq 2$, we use the full power of the primality assumption, which, after Lemma 2 and the subsequent remark, can be read in the following picture:

$$
\begin{aligned}
b_{1} \geq b_{2} \geq \quad \begin{array}{c}
b_{3} \\
\vee
\end{array} & \geq b_{g+1} \geq b_{g+2} \geq b_{g+3} \geq \cdots \geq b_{2 g+1} \\
a_{2 g+1} & \geq \cdots \geq a_{g+3} \geq a_{g+2} \geq a_{g+1} \geq \cdots \geq \quad a_{3} \geq a_{2} \geq a_{1} .
\end{aligned}
$$

Looking at these inequalities, we see that:

if $d \neq g+1$, then

$$
a_{2 g+3-d}=a_{2 g+4-(d+1)}<b_{d+1} \leq v \leq b_{d},
$$

if $d=g+1$ and $a_{g+2} \leq v$, then

$$
a_{2 g+3-d}=a_{g+2} \leq v<b_{d},
$$

if $d=g+1$ and $v<a_{g+2}$, then

$$
b_{d+1}=b_{g+2} \leq v<a_{g+2} .
$$

Hence we have to skip out these two possibilities:

i) $a_{2 g+3-d} \leq v<b_{d}$.

In this case we have

$$
\left\{m \mid b_{m} \leq v\right\}=\{d+1, \ldots, 2 g+1\}
$$

and 


$$
\left\{m \mid a_{m} \leq v\right\} \supseteq\{1,2, \ldots, 2 g+3-d\} .
$$

Hence by $(*)$ we get

$$
0=q_{v} \leq 1+(2 g+1-(d+1)+1)-(2 g+3-d)=-1,
$$

a contradiction.

ii) $b_{g+2} \leq v<a_{g+2}$.

In this case $c-a_{g+2}<a_{g+2}$, hence $a_{g+2}>\frac{c}{2}$. From this we get

$$
a_{g+1}<b_{g+2} \leq v<r \leq\left[\frac{s}{2}\right]+1 \leq \frac{s+2}{2}<\frac{c}{2}<a_{g+2} .
$$

This is absurd because we have no $a_{i}$ 's between $v$ and $r$ so that we cannot pass from $q_{v}=0$ to $q_{r}<0$.

We will prove now the converse. We have a polynomial

$$
h(z)=1+3 z+h_{2} z^{2}+\cdots+h_{s} z^{s} \in \mathbf{Z}[z],
$$

such that $h_{s} \neq 0$ and $h(z)$ verifies conditions a), b) and c) in the theorem. We let

$$
c:=s+3 \quad a:=\min \left\{t \mid h_{t} \neq\left(\begin{array}{c}
t+2 \\
2
\end{array}\right)\right\}
$$

and

$$
\sum_{t=0}^{s+2} q_{t} z^{t}:=h(z)(1-z)^{2}=\Delta^{2} h(z)
$$

We have by b)

$$
q_{t} \leq 0 \quad \text { if } \quad a \leq t \leq\left[\frac{s}{2}\right]+1=\left[\frac{c-3}{2}\right]+1=\left[\frac{c-1}{2}\right]
$$

Since $\Delta^{2} h(z)$ is $(c-1)$-symmetric, we immediately get

$$
q_{t} \leq 0 \quad \text { if } \quad c-1-\left[\frac{c-1}{2}\right] \leq t \leq c-1-a .
$$

But

$$
\left[\frac{c-1}{2}\right] \geq \frac{c-2}{2}
$$

hence 


$$
c-1-\left[\frac{c-1}{2}\right] \leq\left[\frac{c-1}{2}\right]+1
$$

so that we finally get

$$
q_{t} \leq 0, \quad \text { if } \quad a \leq t \leq c-1-a .
$$

Now let

$$
\sum_{i=0}^{c} k_{\imath} z^{i}:=\Delta^{3} h(z)=\Delta\left(\sum_{t=0}^{c-1} q_{t} z^{t}\right)=h(z)(1-z)^{3}
$$

We have already remarked that this polynomial is $c$-antisymmetric. We have some strong informations on its coefficients.

1. $k_{0}=1, k_{c}=-1$.

2. $k_{j}=0$ if $j \in[1, a-1] \cup[c-a+1, c-1]$.

This is easy to see since, by the definition of $a$, for every $i=0, \ldots, a-1$ we have $h_{i}=\left(\begin{array}{c}i+2 \\ 2\end{array}\right)$, hence

$$
\begin{gathered}
h_{i}-h_{i-1}=\left(\begin{array}{c}
i+2 \\
2
\end{array}\right)-\left(\begin{array}{c}
i+1 \\
2
\end{array}\right)=i+1 \\
q_{\imath}=h_{i}-h_{i-1}-\left(h_{\imath-1}-h_{\imath-2}\right)=i+1-i=1
\end{gathered}
$$

and finally

$$
k_{0}=1, \quad k_{i}=q_{i}-q_{i-1}=1-1=0 \text {, for every } i=1, \ldots, a-1 .
$$

The $c$-antisymmetry of $\Delta^{3} h(z)$ gives the conclusion.

3. $k_{a}<0, k_{c-a}>0$.

This is also clear since $k_{a}=q_{a}-q_{a-1}=q_{a}-1$. It follows that $k_{a}<0$ because $q_{a} \leq 0$ by assumption.

Now the crucial remark is that by using the $c$-antisymmetry of $\sum_{\imath=0}^{c} k_{i} z^{\imath}$, we can write in a unique way

$$
\sum_{i=0}^{c} k_{i} z^{i}=1-\sum_{i=1}^{p} z^{a_{i}}+\sum_{i=1}^{p} z^{b_{i}}-z^{c}
$$

where $p, a_{i}$ and $b_{\imath}$ are positive integers such that

$$
b_{i}=c-a_{\imath} \text { for every } i \text { and } a_{i} \neq b_{j} \text {, for every } i, j \text {. }
$$

We may assume that 


$$
a_{1} \leq a_{2} \leq \cdots \leq a_{p}
$$

so that

$$
b_{p} \leq b_{p-1} \leq \cdots \leq b_{1}
$$

Since

$$
\Delta^{3} h(z)=1-\sum_{i=1}^{p} z^{a_{i}}+\sum_{i=1}^{p} z^{b_{i}}-z^{c}
$$

is a multiple of $(1-z)^{3}$, its derivative vanishes at 1 , so that

$$
-\sum_{i=1}^{p} a_{\imath}+\sum_{i=1}^{p} b_{\imath}-c=0
$$

By using the fact that $b_{i}=c-a_{i}$, we get

4. $(p-1) c=2 \sum_{i=1}^{p} a_{i}$.

By 1,2 and 3 above we also get

5. $a_{1}=a, b_{1}=c-a$, and $a_{\imath}, b_{i} \in[a, c-a]$ for every $i=1, \ldots, p$.

Since

$$
\sum_{i=0}^{s+2} q_{i} z^{i}=\frac{1-\sum_{i=1}^{p} z^{a_{i}}+\sum_{i=1}^{p} z^{b_{i}}-z^{c}}{1-z}=\sum\left(1-\sum_{i=1}^{p} z^{a_{i}}+\sum_{i=1}^{p} z^{b_{i}}-z^{c}\right),
$$

we also have

6. $q_{t}=1+\#\left\{m \mid b_{m} \leq t\right\}-\#\left\{m \mid a_{m} \leq t\right\}, \quad t=0 \ldots, c-1$.

We collect some other properties of the integers involved in our computation.

7. $p \geq 2$.

This follows immediately from 4 , since $a_{i} \geq 2$ for every $i$.

8. $a_{2}<b_{p}$, hence $a_{p}<b_{2}$.

If not we have, by $5, a_{1}<b_{p}<a_{2}$, hence

$$
\left\{m \mid a_{m} \leq b_{p}\right\}=\{1\},
$$

and

$$
\left\{m \mid b_{m} \leq b_{p}\right\} \supseteq\{p\},
$$

so that, by 6 , 


$$
q_{b_{p}} \geq 1+1-1=1 \text {. }
$$

Since

$$
b_{2}=c-a_{2}<c-a_{1}=b_{1},
$$

we have

$$
a_{1}<b_{p} \leq b_{2} \leq b_{1}-1=c-a-1 \text {. }
$$

Hence we get

$$
a<b_{p} \leq c-a-1, \text { and } q_{b_{p}} \geq 1,
$$

a contradiction to $\left.b^{\prime}\right)$.

9. $q_{a_{2}}<0$.

By 8 we have

$$
a_{1} \leq a_{2}<b_{p}
$$

hence

$$
\left\{m \mid a_{m} \leq a_{2}\right\} \supseteq\{1,2\}
$$

and

$$
\left\{m \mid b_{m} \leq a_{2}\right\}=\varnothing .
$$

By 6 , this means

$$
q_{a_{2}} \leq 1-2=-1
$$

as wanted.

10. If $p$ is even, then $c$ is even.

This follows immediately from 4 .

11. $a_{2} \leq\left[\frac{s}{2}\right]+1$.

$$
\begin{aligned}
& \text { Otherwise } c-b_{2}>\left[\frac{s}{2}\right]+1 \text {, hence } \\
& \qquad b_{2}<c-\left[\frac{s}{2}\right]-1=s+2-\left[\frac{s}{2}\right] .
\end{aligned}
$$

But, by $8, a_{2}<b_{2}$, hence 


$$
\left[\frac{s}{2}\right]+1<a_{2} \leq b_{2}-1 \leq s-\left[\frac{s}{2}\right]
$$

a contradiction.

12. Let us assume that there exists an integer $t$ such that $a_{2}<t<b_{2}$, and $q_{t}=0$. We claim that this has the following consequences:

12a. $q_{t}=0$ for some integer $t$ such that $a_{2}<t \leq\left[\frac{s}{2}\right]+1$.

If we have

$$
\left[\frac{s}{2}\right]+2 \leq t \leq b_{2}-1=c-a_{2}-1
$$

then we get

$$
a_{2} \leq c-1-t \leq c-1-\left[\frac{s}{2}\right]-2=s-\left[\frac{s}{2}\right] \leq\left[\frac{s}{2}\right]+1 .
$$

Since $0=q_{t}=q_{c-1-t}$ and by $9 q_{a_{2}}<0$, we must have

$$
a_{2}<c-1-t \leq\left[\frac{s}{2}\right]+1
$$

and the conclusion follows

Thank to this last property we may then define the following integer:

$$
n:=\min \left\{t \mid a_{2}<t \leq\left[\frac{s}{2}\right]+1, q_{t}=0\right\} .
$$

12b. $n<c-n<b_{2}$.

Since $n \leq\left[\frac{s}{2}\right]+1$ we have

$$
2 n \leq 2\left[\frac{s}{2}\right]+2 \leq s+2=c-1<c .
$$

On the other hand

$$
c-b_{2}=a_{2}<n
$$

as desired. 
12c. $q_{d}<0$ if $d \in\left[a_{2}, n-1\right] \cup\left[c-n, b_{2}-1\right], q_{d}=0$ if $d \in[n, c-n-1]$.

Since

$$
a \leq a_{2}<n \leq\left[\frac{s}{2}\right]+1
$$

and $q_{a_{2}}<0$ by $9, q_{n}=0$ by assumption, condition c) implies that

$$
q_{d}=0, \quad \text { if } \quad n \leq d \leq\left[\frac{s}{2}\right]+1
$$

From the $(c-1)$-symmetry of $\Delta^{2} h(z)$, we get

$$
q_{d}=0, \quad \text { if } \quad c-2-\left[\frac{s}{2}\right] \leq d \leq c-1-n .
$$

From this we get that $q_{d}=0$ for $n \leq d \leq c-n-1$ since

$$
c-2-\left[\frac{s}{2}\right]=s+1-\left[\frac{s}{2}\right] \leq\left[\frac{s}{2}\right]+2 \text {. }
$$

Moreover, by the true definition of $n$ and the condition $b$ ), it is clear that $q_{d}<$ if $a_{2} \leq d \leq n-1$ and we get the conclusion by the $(c-1)$-symmetry of $\Delta^{2} h(z)$.

12d. For every $i=2, \ldots, p$

$$
a_{\imath}, b_{\imath} \in\left[a_{2}, n\right] \cup\left[c-n, b_{2}\right] .
$$

We know by 8 that $a_{p}<b_{2}$ hence, if $i \geq 2$, we have

$$
a_{2} \leq a_{i} \leq a_{p}<b_{2} \text {. }
$$

But by $12 \mathrm{c}$ we have $q_{n}=\cdots=q_{c-1-n}=0$ hence, by 6 , we cannot have any $a_{i}$ 's or $b_{i}$ 's in the interval $[n+1, c-1-n]$. This gives the conclusion for the $a_{i}$ 's. On the other hand, if $i \geq 2$ we have by 8

$$
a_{2} \leq b_{\imath} \leq b_{2}
$$

and we get the conclusion as before.

13. If $p \geq 4$ then for every $r=3, \ldots,\left[\frac{p}{2}\right]+1$ we have

$$
b_{r}>a_{p+3-r}
$$

If not there exists $r \in\left[3,\left[\frac{p}{2}\right]+1\right]$ such that $b_{r}<a_{p+3-r}$ and we have 


$$
\left\{m \mid b_{m} \leq b_{r}\right\} \supseteq\{r, r+1, \ldots, p\}
$$

and

$$
\left\{m \mid a_{m} \leq b_{r}\right\} \subseteq\{1,2, \ldots, p+2-r\} .
$$

We get by 6

$$
q_{b_{r}} \geq 1+(p-r+1)-(p+2-r)=0 .
$$

Since

$$
a<b_{r}<b_{1}=c-a,
$$

by $\mathrm{b}^{\prime}$ ) we get $q_{b_{r}}=0$ so that

$$
\left\{m \mid a_{m} \leq b_{r}\right\}=\{1,2, \ldots, p+2-r\} .
$$

This implies

$$
a_{p+2-r}<b_{r}<a_{p+3-r} .
$$

Since $q_{b_{r}}=0$ and, by 8 ,

$$
a_{2}<b_{r}<b_{2},
$$

we have the assumption as in 12 . Then by $12 \mathrm{c}$ we get

$$
b_{r} \in[n, c-n-1] \text {, }
$$

while by $12 \mathrm{~d}$

$$
b_{r} \in\left[a_{2}, n\right] \cup\left[c-n, b_{2}\right] .
$$

This implies

$$
b_{r}=n \text {. }
$$

Since by $12 c$

$$
q_{c-n-1}=0, \quad q_{c-n}<0,
$$

we must have $c-n=a_{i}$ for some $i$. But we have

$$
a_{p+2-r}<n<a_{p+3-r},
$$

hence by $12 \mathrm{~d}$ we get

$$
c-n=a_{p+3-r} .
$$


It follows that $a_{p+3-r}=a_{r}$. Since $r \leq\left[\frac{p}{2}\right]+1$ we get $r \leq \frac{p}{2}+1$ which implies $r \leq p+2-r$. Finally we get

$$
a_{r} \leq a_{p+2-r}<a_{p+3-r}=a_{r}
$$

a contradiction. This proves 13

14. Conclusion. We have two possibilities: either $p$ is odd, say $p=2 g+1$, or $p$ is even, say $p=2 g$.

$\mathbf{p}=2 g+1$.

In this case we have $\left[\frac{p}{2}\right]+1=g+1$. Hence, if $g \geq 2$, we may apply 13 to get

$$
b_{r}>a_{p+3-r}=a_{2 g+4-r}, \quad r=3, \ldots, g+1 .
$$

If $p=3$, we certainly have by 8

$$
b_{2}>a_{3}
$$

In any case we have integers

$$
2 \leq a_{1} \leq \cdots \leq a_{2 g+1}
$$

such that by 4 ,

$$
c g=\sum_{i=1}^{2 g+1} a_{i}
$$

Now, if $g=1$, we have $b_{2}>a_{3}$, while, if $g \geq 2$, we have

$$
b_{r}>a_{2 g+4-r}, \quad r=3, \ldots, g+1 \text {. }
$$

As remarked after Lemma 2 , this implies that, in any case, the matrix $\left(u_{i j}:=b_{i}\right.$ $-a_{j}$ ), verifies the conditions $\left.\left.\mathrm{a}\right), \mathrm{b}\right)$ and $\mathrm{c}$ ) in Lemma 1 . Hence we can find a codimension three Gorenstein ideal $I \subseteq R=k[X, Y, Z]$, such that $R / I$ has minimal free resolution

$$
0 \rightarrow R(-c) \rightarrow \bigoplus_{i=1}^{2 g+1} R\left(-b_{i}\right) \rightarrow \bigoplus_{i=1}^{2 g+1} R\left(-a_{i}\right) \rightarrow R \rightarrow R / I \rightarrow 0
$$

This means that 


$$
h(z)=\frac{1-\sum_{i=1}^{2 g+1} z^{a_{i}}+\sum_{i=1}^{2 g+1} z^{b_{i}}-z^{c}}{(1-z)^{3}}
$$

is the $h$-polynomial of $R / I$. By Lemma 3 we get the conclusion.

$\mathbf{p}=2 g$.

Under this assumption we have by 10 that $c$ is even, say

$$
c=2 f \text {. }
$$

We also have

$$
a_{\imath}, b_{\imath} \neq f, \quad i=1, \ldots, p
$$

otherwise, for example, $2 a_{i}=2 f=c=a_{i}+b_{i}$, hence $a_{i}=b_{i}$.

Also it is clear that

$$
a_{2}<f
$$

otherwise $f<a_{2}$ would imply

$$
b_{2}=2 f-a_{2}<a_{2}
$$

a contradiction to 8 .

Let

$$
h:=\max \left\{i \mid a_{i}<f\right\} .
$$

Then $2 \leq h \leq 2 g$. If $h<2 g$, then

$$
a_{h}<f<a_{h+1}
$$

so that

$$
b_{h+1}=2 f-a_{h+1}<f<2 f-a_{h}=b_{h} .
$$

If $h=2 g$, then $a_{2 g}<f$, so that $c-b_{2 g}<f$ which implies

$$
a_{2 g}<f<b_{2 g} \text {. }
$$

We let

$$
a_{j}^{\prime}= \begin{cases}a_{\jmath} & 1 \leq j \leq h \\ f & j=h+1 \\ a_{\jmath-1} & h+2 \leq j \leq 2 g+1\end{cases}
$$

and 


$$
b_{j}^{\prime}= \begin{cases}b_{j} & 1 \leq j \leq h \\ f & j=h+1 \\ b_{j-1} & h+2 \leq j \leq 2 g+1\end{cases}
$$

Then it is clear that we have

$$
a_{1}=a_{1}^{\prime} \leq a_{2}=a_{2}^{\prime} \leq \cdots \leq a_{h}=a_{h}^{\prime}<a_{h+1}^{\prime}=f<a_{h+1}=a_{h+2}^{\prime} \leq \cdots \leq a_{2 g}=a_{2 g+1}^{\prime}
$$

and

$$
b_{1}=b_{1}^{\prime} \geq b_{2}=b_{2}^{\prime} \geq \cdots \geq b_{h}=b_{h}^{\prime}>b_{h+1}^{\prime}=f>b_{h+1}=b_{h+2}^{\prime} \geq \cdots \geq b_{2 g}=b_{2 g+1}^{\prime} .
$$

By 4 with $p=2 g$ and $c=2 f$, we have

$$
(2 g-1) f=\sum_{i=1}^{2 g} a_{\imath}
$$

hence

$$
\sum_{i=1}^{2 g+1} a_{i}^{\prime}=\sum_{i=1}^{2 g} a_{i}+f=(2 g-1) f+f=2 f g=c g .
$$

Further

$$
b_{\imath}^{\prime}=c-a_{i}^{\prime}, \quad i=1, \ldots, 2 g+1 .
$$

Now let $g=1$; then $p=2$ and $h=2$ hence

$$
a_{1}<a_{2}<f<b_{2}<b_{1},
$$

so that

$$
b_{2}^{\prime}=b_{2}>f=a_{3}^{\prime} .
$$

If $g \geq 2$ we claim that

$$
b_{r}^{\prime}>a_{2 g+4-r}^{\prime}, \quad r=3, \ldots, g+1 .
$$

Let us assume by contradiction that $b_{r}^{\prime} \leq a_{2 g+4-r}^{\prime}$ for some $r$ with $3 \leq r \leq g+1$. Then it is clear that

$$
b_{r}^{\prime}<a_{2 g+4-r}^{\prime}
$$

since we can only have equality for $r=h+1=2 g+4-r$. But this would mean $r=2 g+4-r$, so that $r=g+2$, which is absurd.

Since 


$$
g+1=\left[\frac{2 g}{2}\right]+1=\left[\frac{p}{2}\right]+1
$$

we have

$$
3 \leq r \leq\left[\frac{p}{2}\right]+1
$$

We have three possibilities: either $b_{r}^{\prime}>f$ or $b_{r}^{\prime}=f$ or $b_{r}^{\prime}<f$. If $b_{r}^{\prime}>f$ then $b_{r}^{\prime}=b_{r}$ and $a_{2 g+4-r}^{\prime}>b_{r}>f$. This implies

$$
a_{2 g+4-r}^{\prime}=a_{2 g+3-r} \text {. }
$$

Hence

$$
b_{r}<a_{2 g+3-r},
$$

a contradiction to 13 .

If $b_{r}^{\prime}=f$, then

$$
b_{r+1}^{\prime}=b_{r}<f
$$

and

$$
f<a_{2 g+4-r}^{\prime}
$$

hence

$$
a_{2 g+4-r}^{\prime}=a_{2 g+3-r}
$$

This implies

$$
b_{r}<f<a_{2 g+3-r}
$$

which again contradicts 13 .

Finally if $b_{r}^{\prime}<f$ then $b_{r}^{\prime}=b_{r-1}$ and $r \geq h+2 \geq 4$. We have either

$$
b_{r}^{\prime}=b_{r-1}<a_{2 g+4-r}^{\prime}<f
$$

or

$$
b_{r}^{\prime}=b_{r-1}<f<a_{2 g+4-r}^{\prime} .
$$

In any case we get

$$
b_{r-1}<a_{2 g+4-r}=a_{2 g+3-(r-1)} .
$$

Since 


$$
3 \leq r-1 \leq\left[\frac{p}{2}\right]+1
$$

we have again a contradiction to 13 .

The conclusion now follows as in the case $p=2 g+1$ by considering the integers $a_{1}^{\prime}, a_{2}^{\prime}, \ldots, a_{2 g+1}^{\prime}$ instead of $a_{1}, a_{2}, \ldots, a_{2 g+1}$.

Let us consider the 7 -symmetric polynomial

$$
h(z)=1+3 z+4 z^{2}+5 z^{3}+5 z^{4}+4 z^{5}+3 z^{6}+z^{7} .
$$

This is the $h$-polynomial of the codimension three Gorenstein $G$-algebra $R / I$ where

$$
I=\left(Z^{2}, Y Z, Y^{4}-X^{3} Z, X^{4} Y, X^{7}\right)
$$

is the ideal of $R=k[X, Y, Z]$ generated by the pfaffians of the skew-symmetric matrix

$$
\left(\begin{array}{ccccc}
0 & 0 & 0 & X^{3} & Y \\
0 & 0 & X^{4} & Y^{3} & Z \\
0 & -X^{4} & 0 & Z & 0 \\
-X^{3} & -Y^{3} & -Z & 0 & 0 \\
-Y & -Z & 0 & 0 & 0
\end{array}\right)
$$

But we have $a=2, s=7$ so that $\left[\frac{s}{2}\right]+1=4$. Since clearly

$$
\Delta^{2} h(z)=h(z)(1-z)^{2}=1+z-z^{2}-z^{4}-z^{5}-z^{7}+z^{8}+z^{9},
$$

the given polynomial cannot be the $h$-polynomial of a codimension three Gorenstein domain.

Given the polynomial

$$
h(z)=1+3 z+6 z^{2}+10 z^{3}+13 z^{4}+14 z^{5}+14 z^{6}+13 z^{7}+10 z^{8}+6 z^{9}+3 z^{10}+z^{11}
$$

we now explicitly construct a Gorenstein codimension three ideal whose $h$-polynomial is $h(z)$.

We have $a=4$, and $s=11$ so that $\left[\frac{s}{2}\right]+1=6$. We get

$$
\Delta^{3} h(z)=1-2 z^{4}-z^{5}+z^{6}-z^{8}+z^{9}+2 z^{10}-z^{14}
$$


Hence we let

$$
a_{1}=a_{2}=4, a_{3}=5, a_{4}=8
$$

and

$$
b_{1}=b_{2}=10, b_{3}=9, b_{4}=6 .
$$

Since we have $p=4$, we must consider

$$
a_{1}^{\prime}=a_{2}^{\prime}=4, a_{3}^{\prime}=5, a_{4}^{\prime}=7, a_{5}^{\prime}=8
$$

and

$$
b_{1}^{\prime}=b_{2}^{\prime}=10, b_{3}^{\prime}=9, b_{4}^{\prime}=7, b_{5}^{\prime}=6 .
$$

If we let $u_{i j}:=b_{i}-a_{j}$ we get the matrix

$$
\left(\begin{array}{ccccc}
6 & 6 & 5 & 3 & 2 \\
6 & 6 & 5 & 3 & 2 \\
5 & 5 & 4 & 2 & 1 \\
3 & 3 & 2 & 0 & -1 \\
2 & 2 & 1 & -1 & -2
\end{array}\right)
$$

The ideal generated by the pfaffians of the skew-symmetric matrix

$$
\left(\begin{array}{ccccc}
0 & 0 & 0 & X^{3} & Y^{2} \\
0 & 0 & X^{5} & Y^{3} & Z^{2} \\
0 & -X^{5} & 0 & Z^{2} & 0 \\
-X^{3} & -Y^{3} & -Z^{2} & 0 & 0 \\
-Y^{2} & -Z^{2} & 0 & 0 & 0
\end{array}\right)
$$

is the ideal

$$
I=\left(Z^{4}, Y^{2} Z^{2}, Y^{5}-X^{3} Z^{2}, X^{5} Y^{2}, X^{8}\right) .
$$

It is clear that $R / I$ has $h(z)$ as $h$-polynomial. Since $g=2$ and $u_{35}=1>0$, the degree matrix of $I$ verifies the assumptions as in Lemma 3 . Hence we can find a codimension three Gorenstein prime ideal whose $h$-polynomial is $h(z)$.

If we are given a sequence

$$
\left(1,2, c_{2}, \ldots, c_{t}\right)
$$

of non negative integers, we say that it is admissible if the corresponding numeric- 
al function is admissible in the sense we defined before.

By using the classical theorem of Macaulay as in [S], it is easy to see that $\left(1,2, c_{2}, \ldots, c_{t}\right)$ is admissible if and only if for some integer $a \geq 2$ we have

$$
c_{\imath}=i+1, \quad 0 \leq i \leq a-1,
$$

and

$$
c_{i+1} \leq c_{i}, \quad a-1 \leq i \leq t-1 .
$$

Let

$$
h(z)=1+3 z+h_{2} z^{2}+\cdots+h_{s} z^{s} \in \mathbf{Z}[z]
$$

be a $s$-symmetric polynomial. If, as before, $a$ is the initial degree of $h(z)$ and we let

$$
\sum_{i=0}^{t+2} q_{i} z^{i}=\Delta^{2} h(z)=h(z)(1-z)^{2}
$$

the following conditions are equivalent:

a) $q_{\imath} \leq 0$ for every $i \in\left[a,\left[\frac{s}{2}\right]+1\right]$.

b) The sequence $\left(1,2, h_{2}-3, \ldots, h_{\left[\frac{s}{2}\right]}-h_{\left[\frac{s}{2}\right]-1}\right)$ is admissible.

This can be easily proved in the following way.

Since $a$ is the initial degree of $h(z)$, it is clear that

$$
q_{i}=1, \quad i \in[0, a-1],
$$

and

$$
h_{i}-h_{i-1}=\sum_{j=0}^{i} q_{j}, \quad i \geq 1 \text {. }
$$

The result follows easily if we can prove that

$$
h_{\left[\frac{s}{2}\right]}-h_{\left[\frac{s}{2}\right]-1} \geq 0 \Leftrightarrow q_{\left[\frac{s}{2}\right]+1} \leq 0 .
$$

But if $s=2 t+1$, then $h_{\left[\frac{s}{2}\right]+1}=h_{\left[\frac{s}{2}\right]}$, hence

$$
h_{\left[\frac{s}{2}\right]}-h_{\left[\frac{s}{2}\right]-1}=h_{\left[\frac{s}{2}\right]+1}-h_{\left[\frac{s}{2}\right]}-q_{\left[\frac{s}{2}\right]+1}=-q_{\left[\frac{s}{2}\right]+1} .
$$

If $s=2 t$, then $h_{\left[\frac{s}{2}\right]-1}=h_{\left[\frac{s}{2}\right]+1}$, hence

$$
h_{\left[\frac{s}{2}\right]}-h_{\left[\frac{s}{2}\right]-1}=h_{\left[\frac{s}{2}\right]+1}-h_{\left[\frac{s}{2}\right]}-q_{\left[\frac{s}{2}\right]+1}=-\left(h_{\left[\frac{s}{2}\right]}-h_{\left[\frac{s}{2}\right]-1}\right)-q_{\left[\frac{s}{2}\right]+1} \text {, }
$$


and

$$
2\left(h_{\left[\frac{s}{2}\right]}-h_{\left[\frac{s}{2}\right]-1}\right)=-q_{\left[\frac{s}{2}\right]+1} .
$$

In both cases the conclusion follows.

We will say that an admissible sequence $\left(1,2, c_{2}, \ldots, c_{t}\right)$ is of decreasing type if for some integer $b \in[a, t+1]$, we have

$$
c_{i}= \begin{cases}i+1, & i \in[0, a-1] \\ a & i \in[a-1, b-1]\end{cases}
$$

and for $i \in[b-1, t-1]$ either $c_{i}=0$ or $c_{i+1}<c_{i}$.

Proposition 6. The polynomial

$$
h(z)=1+3 z+h_{2} z^{2}+\cdots+h_{s} z^{s}
$$

verifies the conditions a), b) and c) as in Theorem 5 , if and only if $h(z)$ is s-symmetric and the sequence

$$
\left(1,2, h_{2}-3, \ldots, h_{\left[\frac{s}{2}\right]}-h_{\left[\frac{s}{2}\right]-1}\right)
$$

is admissible of decreasing type.

Proof. As before we have

$$
q_{t}=1, \quad t \in[0, a-1]
$$

and

$$
h_{\imath}-h_{i-1}=\sum_{j=0}^{i} q_{j}, \quad i \geq 1 \text {. }
$$

Hence

$$
h_{\imath}-h_{\imath-1}=i+1, \quad i \in[1, a-1] .
$$

Let us assume that the given polynomial verifies the conditions as in Theorem 5 . Then the sequence $\left(1,2, h_{2}-3, \ldots, h_{\left[\frac{s}{2}\right]}-h_{\left[\frac{s}{2}\right]-1}\right)$ is admissible by the preceding remark and we need only to prove that it is of decreasing type.

We have two possibilities.

Case 1. $q_{i}=0$, for every $i \in\left[a,\left[\frac{s}{2}\right]+1\right]$. 
In this case $h_{\imath}-h_{\imath-1}=a$ for every $i \in\left[a-1,\left[\frac{s}{2}\right]\right]$ and the sequence $\left(1,2, h_{2}-3, \ldots, h_{\left[\frac{s}{2}\right]}-h_{\left[\frac{s}{2}\right]-1}\right)$ is of decreasing type.

Case 2. $q_{i}<0$ for some $i \in\left[a,\left[\frac{s}{2}\right]+1\right]$.

In this case let $b$ be the least integer with this property. Then we have

$$
h_{a-1}-h_{a-2}=h_{a}-h_{a-1}=\cdots=h_{b-1}-h_{b-2}=a,
$$

and

$$
h_{b}-h_{b-1}=a+q_{b}<a .
$$

Now, if for some $i \in\left[b,\left[\frac{s}{2}\right]-1\right]$ we have $h_{\imath}-h_{\imath-1}>0$ and $h_{i+1}-h_{\imath}=h_{i}-h_{i-1}$, then $q_{i+1}=0$. By condition c) this implies $q_{j}=0$ for every $j \in\left[i+1,\left[\frac{s}{2}\right]+1\right]$. In turn, this implies

$$
0<h_{i}-h_{\imath-1}=h_{i+1}-h_{\imath}=\cdots=h_{\left[\frac{s}{2}\right]}-h_{\left[\frac{s}{2}\right]-1}=h_{\left[\frac{s}{2}\right]+1}-h_{\left[\frac{s}{2}\right]} .
$$

Now if $s=2 t$, then $h_{\left[\frac{s}{2}\right]-1}=h_{\left[\frac{s}{2}\right]+1}$, hence

$$
h_{\left[\frac{s}{2}\right]}-h_{\left[\frac{s}{2}\right]-1}=h_{\left[\frac{s}{2}\right]-1}-h_{\left[\frac{s}{2}\right]},
$$

which implies

$$
h_{i}-h_{i-1}=h_{\left[\frac{s}{2}\right]}-h_{\left[\frac{s}{2}\right]-1}=0 .
$$

If $s=2 t+1$, then $h_{\left[\frac{s}{2}\right]}=h_{\left[\frac{s}{2}\right]+1}$, which implies

$$
h_{\imath}-h_{i-1}=h_{\left[\frac{s}{2}\right]+1}-h_{\left[\frac{s}{2}\right]}=0 .
$$

In both cases we get the conclusion.

Conversely, let us assume that the sequence $\left(1,2, h_{2}-3, \ldots, h_{\left[\frac{s}{2}\right]}-h_{\left[\frac{s}{2}\right]-1}\right)$ is admissible of decreasing type. If, by contradiction, we have

$$
q_{t}<0, \quad q_{v}=0, \quad q_{r}<0,
$$

with $a \leq t<v<r \leq\left[\frac{s}{2}\right]+1$, then

$$
h_{t}-h_{t-1}<h_{t-1}-h_{t-2} \leq h_{a-1}-h_{a-2}=a \text {. }
$$

Also

$$
h_{v}-h_{v-1}=h_{v-1}-h_{v-2} \text {. }
$$


Since

$$
b \leq t<v<r \leq\left[\frac{s}{2}\right]+1
$$

we get

$$
b-1 \leq v-1 \leq\left[\frac{s}{2}\right]-1
$$

Since the sequence is of decreasing type this means that

$$
0=h_{v-1}-h_{v-2} \text {. }
$$

Hence we get

$$
0=h_{v-1}-h_{v-2} \geq \cdots \geq h_{r-1}-h_{r-2}=h_{r}-h_{r-1}-q_{r}>h_{r}-h_{r-1} \geq 0
$$

a contradiction.

We remark that because of the above proposition, one can see a strong analogy of our result with the characterization of the $h$-polynomial of a perfect codimension two ideal as given by Grouson and Peskine in [GP].

They proved that

$$
1+2 z+h_{2} z^{2}+\cdots+h_{s} z^{s}
$$

is the $h$-polynomial of a codimension two standard Cohen-Macaulay $G$-domain if and only if $h(z)$ is admissible of decreasing type.

\section{REFERENCES}

[BE] Buchsbaum, D., Eisenbud, D., Algebra structures for finite free resolution and some structure theorems for ideals of codimension three, Amer. J. Math., 999 (1977), 447-485.

[GMR] Geramita, A. V., Maroscia, P., Roberts, L., The Hilbert Function of a reduced K-algebra, J. London Math. Soc., 28 (2), n. 3 (1983), 443-452.

[GP] Gruson, L., Peskine, C., Genre des courbes de l'espace projectif, Algebraic Geometry, Lect. Notes Math., 687 (1978), Springer.

[HTV] Herzog, J., Trung, N. G., Valla, G., On hyperplane sections of reduced irreducible varieties of low codimension, (1992), J. Math. Kyoto Univ., 34-1 (1994), 47-72.

[M] Macaulay, F. S., Some property of enumeration in the theory of modular systems, Proc. London Math. Soc., 26 (1927), 531-555.

[S] Stanley, R. P. Hilbert Functions of graded algebras, Adv. in Math., 28 (1978), 57-83.

Dipartimento di Matematica

Università di Genova

Via L. B. Alberti 4

16132 Genova, Italy 